\title{
The impact of natural ventilation on building energy requirement at inter- building scale
}

\author{
Anna Laura Pisello ${ }^{\mathrm{a}, \mathrm{b} *}$, Veronica Lucia Castaldo ${ }^{\mathrm{a}}$, John Eric Taylor ${ }^{\mathrm{c}}$, Franco Cotana $\mathrm{a}^{\mathrm{a}, \mathrm{b}}$ \\ ${ }^{a}$ CIRIAF - Interuniversity Research Center on Pollution and Environment "M. Felli”, University of \\ Perugia, via G. Duranti 63, 06125 Perugia, Italy. \\ ${ }^{\mathrm{b}}$ Department of engineering, University of Perugia, via G. Duranti 93, 06125 Perugia, Italy. \\ ${ }^{\mathrm{c}}$ Charles E. Via, Jr. Department of Civil and Environmental Engineering, Virginia Tech, 114 Patton Hall, \\ Blacksburg, VA 24061, USA.
}

\begin{abstract}
Inter-building effect is responsible for affecting buildings' primary energy requirement for heating, cooling, and lighting. Nevertheless, the impact of natural ventilation should also be considered while predicting building energy demand, since it is documented to substantially affect indoor environmental quality and thermal comfort. This paper investigates the impact of natural ventilation on building primary energy requirement prediction. The Inter-Building Effect (IBE) approach is applied in a typical residential block in Italy. A sensitivity analysis is performed to determine the key input parameter among (i) climate boundary, (ii) infiltration rate, (iii) opening percentage, and (iv) wind strength. Two scenarios, i.e. the stand-alone building and the same building surrounded by its neighborhood, are compared. The thermal-energy dynamic simulation of the different scenarios is carried out to investigate the impact of outdoor airflows on building primary energy requirement for heating and cooling and on indoor thermal comfort, investigated by means of the Thermal Deviation Index method. Inaccuracies in energy need prediction imputable to natural ventilation without taking into account the IBE are detected. The findings show that IBE is much more affected by buildings' opening percentage, infiltration rate, and local wind compared to the weather context since IBE is a local microscale phenomenon.
\end{abstract}

* Corresponding author. Tel.:00390755853796 E-mail address: pisello@crbnet.it (C) 2015 The Authors. 
Keywords: Inter-Building Effect; Natural ventilation; Dynamic thermal-energy simulation; Sensitivity analysis; Building energy performance.

\section{Nomenclature}

SA Stand-alone configuration of the case study building i.e. $1^{\circ}$ case scenario

$\mathbf{N}$ Network configuration of the case study building i.e. $2^{\circ}$ case scenario

IBE first version of Inter-Building Effect accounting for primary energy for heating and cooling

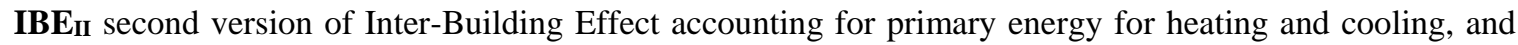
lighting

IP input parameter

OP output parameter

SC sensitivity coefficient

TDI thermal deviation index

TDI $\mathbf{b}_{\text {-s }}$ building thermal deviation index referred to the building performance for each season " $\mathrm{s}$ " (-)

TDI вC-s base case thermal deviation index for each season "s" $\left({ }^{\circ} \mathrm{C} \mathrm{h}\right)$

TDIbsite-s thermal deviation index referred to the building performance freely from the climate (-)

TDI $_{\text {site-s }}$ thermal deviation index for each season s referred to the building in relation to the climate ${ }^{\circ} \mathrm{Ch}$ )

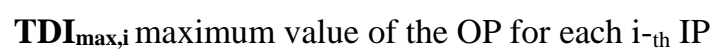

TDI ${ }_{\text {min,i }}$ minimum value of the OP for each $i_{-}$th IP

$\mathbf{T}_{\text {a-s }}$ location sol-air temperature calculated on a reference horizontal surface $\left({ }^{\circ} \mathrm{C}\right)$

To-in indoor thermal zone operative temperature $\left({ }^{\circ} \mathrm{C}\right)$

$\mathbf{T}_{\mathbf{O}, \mathbf{M}-\mathbf{s}}, \mathbf{T}_{\mathbf{O}, \mathbf{m}-\mathrm{s}}$ seasonal target range limits of $\mathrm{T}_{\mathrm{O}-\text { in }}\left({ }^{\circ} \mathrm{C}\right)$

$\mathbf{t}_{\text {s seasonal analysis period }(\mathrm{h})}$

$\mathbf{t}_{\text {T-s }}, \mathbf{t}_{\mathbf{T}-\text {-s,site }}$ periods during which $\mathrm{T}_{\mathrm{O}-\text {-in }}$ and $\mathrm{T}_{\mathrm{a}-\mathrm{s} \text {,site }}$ respectively are within the thermal seasonal target (h)

$\mathbf{T}_{\mathbf{M}-\text { s,site, }} \mathbf{T}_{\mathbf{m}-\text {-s,site }}$ sol-air temperature range limits $\left({ }^{\circ} \mathrm{C}\right)$

$\mathbf{P}_{\mathbf{h}}, \mathbf{P}_{\mathbf{c}}$ periods during which $\mathrm{T}_{\mathrm{O} \text {-in }}$ is out of the seasonal thermal target (h)

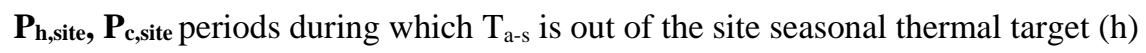

IP generic input parameter

IP $\mathbf{P}_{\text {max, }}$ maximum value of the $i_{\text {-th }}$ IP

IP $P_{\text {min,i }}$ minimum values of the $i_{-}$th IP

$\mathbf{I C}_{\mathbf{i}}$ influence coefficient for each IP

w number of months during which IBE $_{\text {III }}$ is calculated

$\mathbf{P E}_{\mathbf{n}, \mathbf{i}}$ primary energy requirement for cooling and heating of the control building within the surrounding building network for the i-month;

$\mathbf{P E}_{\text {s,i }}$ primary energy requirement for cooling and heating of the stand-alone control building for the i-month 
q volumetric flow through the opening

$\boldsymbol{\delta} \mathbf{P}$ pressure difference across the opening;

n flow exponent varying between 0.5 for fully turbulent flow and 1.0 for fully laminar flow

C flow coefficient, related to the size of the opening

$\mathbf{P}_{\mathbf{w}}$ surface pressure due to wind

$\mathbf{P}$ density of air

$\mathbf{C}_{\mathbf{p}}$ wind pressure coefficient at a given position on the surface

$\mathbf{v}_{\mathbf{z}}$ mean wind velocity at height $\mathrm{z}$

A_min Stand-alone configuration characterized by the lowest IP

A_max Stand-alone configuration characterized by the highest IP

N_min Network of Buildings configuration characterized by the lowest IP

N_max Network of Buildings configuration characterized by the highest IP

\section{Introduction and Research Background}

Climate change and global warming phenomena represent a mounting threat to peoples' health and life, since they lead to increased air temperatures and consequently higher $\mathrm{CO}_{2}$ emissions in the atmosphere [1-3]. It is largely acknowledged that almost $60 \%$ of the global energy consumption in developed countries is attributable to buildings, and that cities are responsible for up to $80 \%$ of the global $\mathrm{CO}_{2}$ emissions [5-6]. Therefore, the necessity to implement innovative low-cost and sustainable technologies for the design of more efficient and comfortable buildings is now undeniable, since local climate change will lead to even higher building energy consumption and greenhouse gas emissions [7-9]. In response to these trends, during the last decade a substantial research effort has been dedicated to the development of strategies to reduce building energy consumption. The same strategies were frequently investigated for being able to mitigate localized overheating. For instance, the use of high albedo materials for urban surfaces [10] was demonstrated to be one of the most effective solutions to decrease cooling energy consumption in summer and to reduce urban overheating, together with the increase of vegetated urban surface [11-12], able to reduce urban heat island at the inter-building level and energy needs in both summer and winter conditions.

Along the same lines, the optimized design and exploitation of natural ventilation among buildings located in close proximity in dense urban environments has been demonstrated to play a key role for (i) improving indoor thermal comfort, (ii) reducing energy requirements, and (iii) mitigating urban heat islands [13]. In fact, it was demonstrated that the lack of control of natural ventilation in buildings leads to the reduction of occupants' thermal comfort [4].

A good understanding and prediction of natural ventilation phenomena and infiltration rates in buildings and its relationship with the outdoor local microclimate can help to resolve many issues related to indoor environmental quality [14]. In particular, night cooling as a combination of natural and mechanical night 
ventilation was found to be effective in the reduction of cooling load [15]. Artmann et al. [16], for instance, managed to map regions with sufficient night cooling potential to define a sort of climate cooling potential index based on the indoor-outdoor temperature gap. Moreover, many numerical tools for wind-driven ventilation design have been proposed, in order to effectively predict external pressure coefficients and airflow rate (e.g., [17]). Furthermore, the possibility to apply controlled natural ventilation as a natural cooling ventilation technique was studied by Chiesa and Grosso [18] with reference to the Mediterranean area. In this study, the complex relationship between local climate phenomena, building characteristics, and ventilative cooling was assessed. Natural ventilation has been therefore demonstrated to be one of the key design parameters affecting the buildings' indoor thermal behavior [19], since a correct airflow rate from the outside can be used to maximize the annual thermal comfort level within occupied spaces. A detailed analysis of the transmission losses and gains for investigating the potential of natural ventilation was performed by Andelkovic et al. [20]. It was demonstrated that optimized night-time natural ventilation regimes and airflow rates can help reduce the overall energy consumption by preventing overheating in summer in the case of a multi-story building located in Belgrad (Serbia), with a naturally ventilated double-skin facade. In this same scenario, full-scale experiments to determine the airflow rate in naturally ventilated rooms of a building located in Corsica (France), were carried out in cross ventilation configurations in order to develop different airflow modelling approaches [21]. The coupling between thermal models was found out to be suitable for model based natural ventilation control.

In particular, the effectiveness of such ventilative cooling action is strongly affected by buildings' thermal characteristics and climate boundary conditions in terms of wind speed, wind direction, and outdoor air temperature $[22,23]$. In fact, a non-negligible effect on building energy requirements is produced by a building's surroundings affecting the thermal-energy performance of the building and microclimate conditions [24]. This phenomenon has been defined as the Inter-Building Effect (IBE) [25]. The IBE is an index aimed at quantifying the effect of the mutual interaction of adjacent buildings in terms of year-round energy performance. The purpose of the IBE is to determine the inaccuracy in energy predictions deriving from considering the building as a non-realistic stand-alone entity rather than surrounded by its real urban context [26]. In fact, non-negligible impact on buildings' heating and cooling requirement was previously detected [24-25] due to the mutual interaction among buildings. In particular, inaccuracies in energy requirement estimation up to $42 \%$ were found in summer in Miami (FL) through the application of IBE method. Therefore, the presence of surrounding buildings cannot be neglected while simulating buildings' energy need in urban environment by means of dynamic simulation tools. Moreover, significant inaccuracies in the primary energy requirement prediction were detected when lighting is not taken into account [27]. This results in the necessity to include lighting in energy simulations as it has a significant influence on building primary energy requirement at the inter-building scale. Lastly, recent studies have examined the impact of IBE on localized heating of building surfaces with implications for Urban Heat Island effects [28]. 
Although Inter-Building Effect research has examined from many perspectives as described in the previous paragraphs, research is lacking that would consider the significant impact on building energy behavior at urban scale that could be created by natural ventilation [29, 30]. In fact, both the airflow and flow regime in an urban environment can be significantly modified and affected by the presence of buildings located in mutually close proximity, acting as obstacles and "shields" to the air flow. Therefore, the lack of consideration of the urban surrounding might generate errors and inaccuracies in the primary energy prediction also due to the different natural ventilation phenomena caused by the presence of surrounding buildings.

Natural ventilation is produced by two main forces; (i) stack effects and (ii) wind. It depends therefore on both geometry parameters such as building size, shape, position, and orientation, and on meteorological factors i.e. wind-speed and outdoor air temperatures. For this reason, accurate airflow analyses are due while predicting building thermal-energy behavior within dynamic simulation tools [31]. To accurately predict the impact of natural ventilation and airflows among buildings in urban environments, a combination of experimental approaches and Computational Fluid Dynamic (CFD) simulation is required [32-36]. Furthermore, the integration of Air Flow Network (AFN) models with Building Energy Simulation tools (BES) is of great interest for the proper numerical analysis of airflows [37, 38], as ventilation effects on building energy requirement depend on several factors, i.e. local climate boundary conditions, thermal mass, operational parameters, and convective heat transfer conditions. Additionally, sensitivity analyses [39, 40] are needed when dealing with such complex phenomena, in order to determine the mutual impact of different input simulation parameters on building performance.

\section{Purpose of the work}

While previous studies were mainly focused on the performance prediction of natural ventilation in terms of thermal comfort and passive cooling capability, the present research seeks to investigate the impact of natural ventilation phenomena on Inter-Building Effect in terms of building primary energy requirement for heating and cooling and thermal comfort conditions. Therefore, starting from previous works on the Inter-Building Effect (IBE) analyses [24-28], the energy performance prediction of two different case scenarios is performed in a dynamic simulation environment [41]: (i) a single residential building considered as stand-alone (Astand-alone configuration-scenario 0) and (ii) the same building surrounded by the adjacent buildings of its real neighborhood (N-network configuration-scenario 1).

To this aim, a preliminary sensitivity study is carried out to determine the main input parameters affecting the IBE in terms of building thermal performance when considered as a single entity (A-scenario 0) rather than inside its realistic urban context (N-scenario 1). Therefore, the influence of different input parameters (IP) is investigated through the dynamic simulation of several case scenarios, in both the assessed configurations i.e. A and $\mathrm{N}$ configurations. In particular, the IP variables selected for the sensitivity analysis 
purpose are (i) climate boundary condition, (ii) infiltration rate, (iii) opening percentage on building façade, and (iv) wind strength.

Finally, the Inter-Building Effect approach [24] is applied to a typical Italian residential block to quantify the differences in primary energy requirement imputable to the modified natural ventilation deriving from the consideration of the building as stand-alone rather than surrounded by its real urban context.

Building upon previous researches aiming at studying the Inter-Building Effects, the original contribution of the present work is therefore represented by the integration into the IBE approach of the natural ventilation phenomena. The main purpose is therefore to quantify the inaccuracies in the primary energy requirements prediction attributable to wind effect and its impact in terms of IBE.

\section{Materials and Method}

The methodology applied in this research consists of the following main steps:

- selection of the case study buildings, i.e. suburban residential block in central Italy;

- sensitivity analysis to evaluate the main parameter affecting the IBE in terms of building thermal performance;

- dynamic energy simulation of the energy performance of the case study in the two considered scenarios i.e. (i) stand-alone building and (ii) building within its real urban context;

- post-processing of the results in terms of IBE, by analyzing the difference between the two evaluated scenarios attributable to the natural ventilation in terms of primary energy need for heating and cooling;

- discussion of the results and main conclusions.

\subsection{Sensitivity Analysis}

The first phase of the study consists of the sensitivity analysis [38] aimed at quantifying the output variation (OP) in terms of TDI i.e. Thermal Deviation Index [40] related to the variation of specifically selected input parameters (IP). TDI is a non-dimensional objective function defining building thermal performance in freefloating conditions. It represents the gap between the output simulated conditions and the thermal target [4043] expressed in terms of indoor operative temperature. The seasonal ranges for summer-winter analysis are defined according to the EN 15251 European Standard [44]. Therefore, the lower the TDI value, the better the indoor thermal performance is expected to be. For instance, a TDI higher than 1 means that the indoor thermal conditions are far from the target.

The TDI ${ }_{b-s}$ or Building Thermal Deviation Index for each season " $\mathrm{s}$ " is expressed in the following way:

$T D I_{b-s}=\frac{\int_{P h}\left[f\left(T_{o-i n}\right)-T_{o, M-s}\right] d t+\int_{P C}\left[f\left(T_{o, m-s}\right)-T_{o-i n}\right] d t}{T D I_{B C-s}} \cdot \frac{t_{s}-t_{T-s}}{t_{s}}$

Where:

- $\mathrm{T}_{\mathrm{O} \text {-in: }}$ indoor operative temperature, calculated in the center of each thermal zone; 
- $\mathrm{T}_{\mathrm{O}, \mathrm{M}-\mathrm{s}}$ : highest value of the indoor operative temperature target range;

- $\mathrm{T}_{\mathrm{O}, \mathrm{m}-\mathrm{s}}$ : and lowest value of the indoor operative temperature target range;

- $\mathrm{T}_{\mathrm{s}}$ : seasonal period of the analysis;

- $\mathrm{T}_{\mathrm{T}-\mathrm{s}}$ : period during which TO-in is included within the thermal seasonal target $23-26^{\circ} \mathrm{C}$, maximum and minimum values considered for summer conditions;

- $\mathrm{TDI}_{\mathrm{BC}-\mathrm{s}}$ : arbitrary base case scenario represented by a constant operative indoor temperature $3^{\circ} \mathrm{C}$ far from each seasonal target;

- $\mathrm{P}_{\mathrm{h}}, \mathrm{P}_{\mathrm{c}}$ : integration domains.

The $\mathrm{TDI}_{\mathrm{b}-\mathrm{s}}(1)$ can be therefore defined as a product of (i) the ratio between the sum of the areas in which the $\mathrm{T}_{\mathrm{O} \text {-in }}$ is out of the thermal target and a base case index $\mathrm{TDI}_{\mathrm{BC}-\mathrm{s}}$, and (ii) the frequency of the $\mathrm{T}_{\mathrm{O} \text {-indoor }}$ distance from the indoor seasonal thermal target, which varies between 0 and 1 [42].

In particular, the $\mathrm{TDI}_{\mathrm{BC}-\mathrm{s}}$ for seasonal analysis is calculated as follows:

$T D I_{B C-s}=\int_{t_{s}}\left[\left(T_{O, M-s}+3\right)-T_{O, M-s}\right] \partial t=3 \cdot t_{s} \quad\left[{ }^{\circ} \mathrm{Ch}\right]$

The integration domains $\mathrm{P}_{\mathrm{h}}$ and $\mathrm{P}_{\mathrm{c}}$ in (1) are defined as follows:

$P_{h}=\left\{\tau \in\left[0, t_{s}\right]: f\left(T_{o-i n}\right) \geq T_{O, M-s}\right\} \quad[\mathrm{h}]$

$P_{c}=\left\{\tau \in\left[0, t_{s}\right]: f\left(T_{o-i n}\right) \leq T_{O, M-s}\right\} \quad[\mathrm{h}]$

This index can be very useful to evaluate the thermal performance of buildings simulated in free floating conditions, and to express how different building parameters can influence the thermal performance.

In order to evaluate a building's thermal performance in relation to specific climate boundary and weather site conditions, the $\mathrm{TDI}_{\text {site, }}$ is defined, as follows:

$T D I_{\text {site }, s}=\left\{\int_{P_{h, s i t e}}\left[f\left(T_{a-s}\right)-T_{M-s, s i t e}\right] \delta \tau+\int_{P_{c, s i t e}}\left[T_{m-s, s i t e}-f\left(T_{a-s}\right)\right] \partial \tau\right\} \cdot \frac{t_{s}-T_{T-s i t e, s}}{t_{s}}\left[{ }^{\circ} \mathrm{Ch}\right]$

Where:

- $T_{\text {a-s: }}$ location sol-air temperature calculated on a reference horizontal surface;

- $\mathrm{T}_{\mathrm{M}-\mathrm{s}, \text { site, }} \mathrm{T}_{\mathrm{m}-\mathrm{s}, \mathrm{site}}$ : extreme values of the seasonal thermal ranges, expressed by $\mathrm{T}_{\mathrm{a}-\mathrm{s}, \mathrm{site}}$. This reference thermal range is an extension of the internal range on the hotter and colder temperature for $3^{\circ} \mathrm{C}$.

The complex index $\mathrm{TDI}_{\mathrm{b} \text {,site }}$ to define the building global performance freely from the location climate conditions is therefore calculated as follows for the seasonal period "s":

$T D I_{b, \text { site-s }}=\frac{\left\{\int_{P_{h}}\left[f\left(T_{o-i n}\right)-T_{O, M-s}\right] \partial \tau+\int_{P_{C}}\left[T_{O, M-s}-f\left(T_{o-i n}\right)\right] \partial \tau\right\} \cdot \frac{t_{s}-t_{T-s}}{T_{s e a s}}}{\left\{\int_{P_{h-s i t e}}\left[f\left(T_{a-s, s i t e}\right)-T_{M s, \text { site }}\right] \partial \tau+\int_{P_{c-s i t e}}\left[T_{m-s, s i t e}-f\left(T_{a-s, s i t e}\right)\right] \partial \tau\right\} \cdot \frac{t_{s-t} t_{T-s, \text { site }}}{T_{S}}}$

where $\mathrm{P}_{\mathrm{h}, \text { site }}$ and $\mathrm{P}_{\mathrm{c}, \text { site }}$ typical of the location are the time periods during which the $\mathrm{T}_{\mathrm{a}-\mathrm{s} \text {,site }}$ is external to the thermal target range: 
$P_{h, \text { site }}=\left\{\tau \in\left[0, t_{s}\right]: f\left(T_{a-s, \text { site }}\right) \geq T_{M-s, \text { site }}\right\}$

$P_{c, \text { site }}=\left\{\tau \in\left[0, t_{s}\right]: f\left(T_{a-s, s i t e}\right) \leq T_{M-s, \text { site }}\right\}$

[h]

The further $\mathrm{TDI}_{\mathrm{b} \text {,site }}$ is from zero, the more the thermal zone registers thermal conditions far from the target. In the present sensitivity analysis, the selected IPs are:

- the climate weather file $(\mathrm{C})$;

- the infiltration level (I);

- the opening percentage on building facades $(\mathrm{O})$;

- the wind strength $(\mathrm{W})$.

The variability of each selected IP is summarized in Table 1:

\begin{tabular}{lccc}
\hline Input parameters (IP) & \multicolumn{3}{c}{ Levels } \\
\cline { 2 - 4 } & Low & Medium & High \\
\hline C-Climate weather file & C_a & C_b & C_c \\
& 0-Trieste & 1-Pisa & 2 -Brindisi \\
\hline I-Infiltration rate [vol/h] & I_a & I_b & I_c \\
& 0.3 & 0.5 & 0.7 \\
\hline O-Opening [\%] & O_a & O_b & O_c \\
& 15 & 30 & 45 \\
\hline W-Wind strength [m/s] & W_a & W_b & W_c \\
& 0-weak & 1-medium & 2-strong \\
\hline
\end{tabular}

Table 1. Variability of each IP for the sensitivity analysis purpose.

The choice of the input parameters (IP) is related to realistic mean and extreme conditions of Italian climate conditions in terms of wind force and characteristic residential construction according to [43-46]. All the input parameters of the numerical simulations have been assumed and varied by considering the indications of the local regulations $[43,44,48,49,51]$. In particular, as concerning the climate IP, the TMY weather files [55-56] of three different Italian locations are selected to be used for the analysis: a cold-temperate Italian climate, i.e. Trieste (Northern Italy), a temperate climate i.e. Pisa (Central Italy), and a hot-temperate climate, i.e. Brindisi (Southern Italy). These locations are selected as the meteorological monitoring stations are all located close to the sea and at the same altitude above sea level, therefore the measurements are comparable since they are taken in consistent conditions. The infiltration rate values are defined in terms of air changes per hour (ac/h), i.e. zone volume per hour under normal operating pressures, and are selected by considering typical values suggested by the local regulations for continuously occupied residences [43], i.e. 0.3, and by varying them between maximum and minimum values as indicated by the local standards [48-51]. The opening percentage was assumed and varied on the real typical construction and geometrical characteristics of Italian residential buildings. As for the wind intensity, the three different IPs are obtained by proportionally increasing and decreasing the start wind velocity value selected as representative of the base case scenario, 
i.e. Pisa wind velocity. Further details about the wind values used for the dynamic simulations are provided in section 4.2 .

The aim of the sensitivity analysis [50-52] is to identify which is the main input parameter affecting the simulation output in terms of building thermal performance, through an IBE perspective. In particular, TDI is the multivariable function depending to the different IP i.e. climate boundary conditions (TMY weather file) $[55,56]$, infiltration rate $[\mathrm{vol} / \mathrm{h}]$, opening percentage on the façade [\%], and wind strength $[\mathrm{m} / \mathrm{s}]$. Therefore, this analysis is aimed at investigating if such IPs equally affect the TDI (OP) with varying the modeling configuration, i.e. A (scenario 0 ) and $\mathrm{N}$ (scenario 1). Therefore, the models elaborated for the sensitivity analysis are summarized in Table 2 :

\begin{tabular}{|c|c|c|}
\hline $\begin{array}{l}\text { Modelled } \\
\text { scenarios }\end{array}$ & $\begin{array}{l}\text { A-Stand-alone configuration } \\
\text { building as stand-alone entity }\end{array}$ & $\begin{array}{l}\text { N-Network configuration } \\
\text { building surrounded by its neighborhood }\end{array}$ \\
\hline & A_Ca-Climate weather file: Trieste & N_Ca-Climate weather file: Trieste \\
\hline & A_Cb-Climate weather file: Pisa & N_Cb-Climate weather file: Pisa \\
\hline & A_Cc-Climate weather file: Brindisi & N_Cc-Climate weather file: Brindisi \\
\hline & A_Ia-Infiltration rate: $0.3 \mathrm{vol} / \mathrm{h}$ & N_Ia-Infiltration rate: $0.3 \mathrm{vol} / \mathrm{h}$ \\
\hline & A_Ib-Infiltration rate: $0.5 \mathrm{vol} / \mathrm{h}$ & $\mathbf{N} \_$Ib-Infiltration rate: $0.5 \mathrm{vol} / \mathrm{h}$ \\
\hline & A_Ic-Infiltration rate: $0.7 \mathrm{vol} / \mathrm{h}$ & N_Ic-Infiltration rate: $0.7 \mathrm{vol} / \mathrm{h}$ \\
\hline & A_Oa-Opening percentage: $15 \%$ & N_Oa-Opening percentage: $15 \%$ \\
\hline & A_Ob-Opening percentage: $30 \%$ & N_Ob-Opening percentage: $30 \%$ \\
\hline & A_Oc-Opening percentage: $45 \%$ & N_Oc-Opening percentage: $45 \%$ \\
\hline & A_Wa- Wind strength: weak & N_Wa- Wind strength: weak \\
\hline & A_Wb- Wind strength: medium & N_Wb- Wind strength: medium \\
\hline & A_Wc- Wind strength: strong & N_Wc- Wind strength: strong \\
\hline
\end{tabular}

Table 2. Summary of the different case scenarios examined for the sensitivity analysis purpose.

The main purpose of the present sensitivity analysis is to perform a sort of input-output analysis for analyzing data from the dynamic simulation procedure [51,52], by quantitatively comparing the output OP (i.e. TDI) variation with respect to the corresponding input parameters IPs' variation, consisting of both climate boundaries (" $\mathrm{C}_{\mathrm{i}}$ " and " $\mathrm{W}_{\mathrm{i}}$ ") and construction features and geometrical characteristics of the case study building (" $\mathrm{I}_{\mathrm{i}}$ ” and " $\mathrm{O}_{\mathrm{i}}$ ").

The influence coefficients $\mathrm{IC}_{\mathrm{i}}$ of the different IPs, calculated as the ratio between the change of output TDI with respect to the change of the corresponding i-th IP, is described by equations 9-12:

$$
\begin{aligned}
& I C_{C}=\frac{\partial T D I}{\partial C_{i}} \approx \frac{\Delta T D I}{\Delta C_{i}} \\
& I C_{W}=\frac{\partial T D I}{\partial W_{i}} \approx \frac{\Delta T D I}{\Delta W_{i}} \\
& I C_{I}=\frac{\partial T D I}{\partial I_{i}} \approx \frac{\Delta T D I}{\Delta I_{i}}
\end{aligned}
$$


$I C_{o}=\frac{\partial T D I}{\partial O_{i}} \approx \frac{\Delta T D I}{\Delta O_{i}}$

Since the realistic quantification of the thermal comfort in terms of TDI depends on multiple factors concerning climate boundary, building characteristics etc., the output result could be expressed as a multivariable function $f$ of $n$ variables i.e. input parameters $\mathrm{IP}_{\mathrm{i}}$ as follows:

$T D I=f\left(I P_{1}, I P_{2}, \ldots ., I P_{n}\right)$

By expressing Eq. (13) as a function of the input parameters of interest in this work, Eq. (13) can be written as follows (14):

$T D I=f(C, W, I, O)$

Where:

- $\mathrm{C}$ is the considered climate boundary conditions;

$-\mathrm{W}$ is the wind strength;

- I is the infiltration rate;

- $\mathrm{O}$ is the opening percentage on buildings' façade;

- TDI is the thermal deviation index representing our output parameter.

Therefore, by considering the IPs selected in this work, the differential of TDI is calculated by means of the chain rule of partial differentiation:

$d T D I=\frac{\partial T D I}{\partial C} \partial C+\frac{\partial T D I}{\partial W} \partial W+\frac{\partial T D I}{\partial I} \partial I+\frac{\partial T D I}{\partial O} \partial O$

Then, by assuming that the considered input parameters (IP) are mutually independent, the partial derivatives of the IPs may be approximated to its simple differential and eq. (16) is verified. By substituting eq. (16) in (15), we obtain Eq. (17).

$$
\begin{aligned}
& \frac{d W}{d C}=\frac{d I}{d C}=\frac{d O}{d C}=0 \\
& \frac{d T D I}{d C}=\frac{\partial T D I}{\partial C}
\end{aligned}
$$

Therefore, the sensitivity coefficient $\mathrm{SC}_{\mathrm{I}}$ of each considered parameter $\mathrm{IP}_{\mathrm{i}}$, (such as " $\mathrm{C}$ " chosen as example in Eq. 16-17) can determined by varying one parameter at a time (OAT method). The range of variability for the IPs (Table 1) is previously defined for each parameter. More in details, the Sensitivity Coefficients SC, defining the impact of each $\mathrm{IP}_{\mathrm{i}}$ on building thermal performance expressed in terms of TDI [38], are calculated as follows:

$$
S C_{I P_{i}}=\frac{\frac{T D I_{\max , i}-T D I_{\min , i}}{T D I_{\max , i}}}{\frac{I P_{\max , i}-I P_{\min , i}}{I P_{\max , i}}}[-]
$$




\section{Where:}

- TDI $_{\max , i}$ : maximum value of the OP for each $\mathrm{i}_{\text {-th }} \mathrm{IP}$;

- TDI ${ }_{\text {min,i: }}$ minimum value of the OP for each $\mathrm{i}_{\text {-th }}$ IP;

- IP $\mathrm{P}_{\mathrm{i}}$ generic input parameter;

- IP max,i $_{\text {: }}$ maximum value of the $\mathrm{i}_{\text {-th }}$ IP;

- IP ${ }_{\text {min, }, \mathrm{i}}$ : minimum values of the $\mathrm{i}_{\text {-th }}$ IP.

\subsection{The Inter-Building Effect approach}

The Inter-Building Effect (IBE) analysis is carried out in order to quantify the mutual impact of buildings situated in close proximity of other buildings, in terms of building primary energy requirement. The further the IBE value is from zero, the greater the inaccuracy in the prediction of the building energy requirement. In this paper, the IBE approach is expanded to include the differences in the prediction of the "control building" primary energy need directly imputable to the variation of natural ventilation and airflows generated by the presence of surrounding buildings.

To this aim, the year-round dynamic energy simulation of the following scenarios is performed:

- the control building as stand-alone object (A);

- the control building within the network of surrounding buildings $(\mathrm{N})$.

For each scenario, the results are post-processed in terms of primary energy requirement for heating and cooling $\left[\mathrm{kWh} / \mathrm{m}^{2}\right]$.

The Inter-Building Effect (IBE $\mathrm{III})$ index is calculated as follows:

$I B E=\frac{\sum_{i=1}^{n}\left(P E_{n, i}-P E_{S, i}\right)}{P E_{S, i}} * 100$

where:

- n: number of months during which IBE is calculated;

- $\mathrm{PE}_{\mathrm{n}, \mathrm{i}}$ : primary energy requirement for cooling and heating of the control building within the surrounding building network for the i-month;

- $\mathrm{PE}_{\mathrm{s}, \mathrm{i}}$ : primary energy requirement for cooling and heating of the stand-alone control building for the imonth.

The calculated values of IBE represent the inaccuracy level in terms of overestimation or underestimation of the building energy need. The main limitation of such approach is the strong dependence on the building geometry and on the configuration of the urban environment surrounding the control building selected as case study. 


\subsection{Numerical analysis}

The energy analysis in terms of IBE is performed by means of EnergyPlus, a dynamic energy simulation tool. In particular, a sophisticated analysis of convective thermal exchanges including natural ventilation is performed, in order to investigate its effect in terms of IBE. In fact, building geometry and surroundings configuration play a major role in determining wind effects, as the presence of close buildings could deflect wind along surfaces and modify the airflow pattern, thereby affecting building thermal-energy performance. Such phenomena cannot be taken into account in the "simple ventilation option" implemented in Design Builder, where airflow and infiltration levels are arbitrarily assumed by the user and defined directly as "scheduled" air changes rate per hour. Therefore, a more detailed natural ventilation simulation method is used here, therefore the flow rates are not arbitrarily assumed but are calculated within the simulation based on the size, configuration, position, and orientation of openings and cracks in the building fabric as well as the simulated wind-speed, pressure, inside and outside temperatures, etc.

In particular, the pressure caused by wind blowing on an external surface is calculated using (i) wind data from the climate weather file and (ii) the wind pressure coefficient for current particular wind incidence angle on building surface. Such wind incidence angles change whether or not surrounding buildings of the urban environment are considered in the numerical analysis.

Therefore, the input data for the simulations in terms of infiltration rate [vol/h], were determined based on the indications of the local regulation UNI TS 11300 [43] and were varied according to the indications of the other local standards [44, 48, 49, 53]. Additionally, the wind speed parameters were determined by proportionally varying the wind intensity values starting from the Pisa TMY weather file (i.e. medium wind) in order to generate two other weather files representative of (i) a strong wind and (ii) a weak wind condition (see section 4.1).

In this view, the energy performance prediction performed by taking into account natural ventilation phenomena could be strongly affected by the IBE. In fact, the ventilation rate (q) through each opening in the building model is calculated based on the pressure difference using wind and stack pressure effects as in the following equation:

$q=C \times \Delta(P)^{n}$

where:

- q: volumetric flow through the opening;

- $\Delta(\mathrm{P})$ : pressure difference across the opening;

- n: flow exponent varying between 0.5 for fully turbulent flow and 1.0 for fully laminar flow;

- C: flow coefficient, related to the size of the opening.

While the flow coefficient $C$ is related to the characteristics of the single "control" building selected as case study, all the other parameters in equation (4) i.e. $\mathrm{q}, \delta \mathrm{P}$, and $\mathrm{n}$ are influenced by urban configuration and 
therefore they change whether the control building is considered as a stand-alone entity rather than a surrounded by a network of buildings.

The pressure on any point on the surface of a building facade can be represented by the following equation:

$P_{w}=0.5 \cdot \rho \cdot C_{p} \cdot v_{z}^{2}$

where:

- $\mathrm{P}_{\mathrm{w}}$ : surface pressure due to wind;

- $\rho$ : density of air;

- $\mathrm{C}_{\mathrm{p}}$ : wind pressure coefficient at a given position on the surface;

- $\mathrm{v}_{\mathrm{z}}$ : mean wind velocity at height $\mathrm{z}$.

While $\rho$ is a constant parameter, $v_{z}$ (eq. 21) is variable depending on the urban shape, urban density, and spatial configuration around the "control" building selected for the analysis of the surface pressure on the façade. Therefore, it changes whether adjacent buildings are considered or not into the dynamic simulation process.

Additionally, the wind pressure coefficient, $\mathrm{C}_{\mathrm{p}}$, is a function of wind direction, of position on the building surface, and, above all, of side exposure. The side exposure varies depending on the degrees of shelter and wind directions and, consequently, it changes whether the surrounding urban context is taken into account or not into the analysis.

\subsection{Description of the Case study}

A typical suburban block in central Italy is selected as case study. This choice was made in order to make the analysis as representative as possible of the majority of the residential buildings in Italy, in order to have the possibility to extend the results of the present analysis to the Italian building stock. To this aim, a standard and 'prototypical' building $(16 \times 10 \times 14 \mathrm{~m})$ was designed, by following the traditional construction characteristics of Italian residential buildings in terms of materials' types, materials' thermal properties, geometry, HVAC energy systems according to previous studies about the features of Italian buildings, as described within the framework of previous European projects [54].

After modeling all the buildings within one block, a "control building" is selected for the analysis of the InterBuilding Effect attributable to natural ventilation, in terms of primary energy requirement for heating and cooling. The "control building" is a typical Italian residential three story building (Figure 1).

Fig. 1(a, b). Picture of the residential building selected as case study in the Stand-Alone configuration (A) and Network (N), respectively (from Design Builder). 
The structural system consists of reinforced concrete columns and beams. The opaque envelope consists of external brickwork $(0.10 \mathrm{~m})$, XPS insulation panel $(0.08 \mathrm{~m})$, concrete block $(0.10 \mathrm{~m})$ and internal gypsum plasterboard $(0.013 \mathrm{~m})$, with a global thermal transmittance of $0.35 \mathrm{~W} / \mathrm{m}^{2} \mathrm{~K}$. The internal partitions are made of two layers of gypsum plasterboard $(0.025 \mathrm{~m})$ with an internal air gap $(0.10 \mathrm{~m})$. The roof is composed by an internal roofing felt $(0.005 \mathrm{~m})$, stone wool insulation $(0.24 \mathrm{~m})$, and clay tile $(0.025 \mathrm{~m})$, and it presents a global thermal transmittance of $0.16 \mathrm{~W} / \mathrm{m}^{2} \mathrm{~K}$. The ground floor consists of cast concrete slab $(0.10 \mathrm{~m})$, XPS insulation layer $(0.11 \mathrm{~m})$, fiberboard $(0.013 \mathrm{~m})$, and asphalt $(0.09 \mathrm{~m})$, for a global thermal transmittance of $0.25 \mathrm{~W} / \mathrm{m}^{2} \mathrm{~K}$. The external windows have double clear glass panes ( $3 \mathrm{~mm}-3 \mathrm{~mm}$ with $13 \mathrm{~mm}$ air) with internal venetian blinds and a wood frame with no thermal break. Table 3 summarizes the main characteristics of the case study building components:

\begin{tabular}{|c|c|c|c|c|}
\hline $\begin{array}{l}\text { Building } \\
\text { component }\end{array}$ & Layers & Thickness [m] & $\begin{array}{l}\text { Conductivity } \\
{\left[\mathbf{m}^{2} \mathbf{K} / \mathrm{W}\right]}\end{array}$ & $\begin{array}{l}\text { Thermal } \\
\text { transmittance } \\
{\left[\mathrm{W} / \mathbf{m}^{2} \mathbf{K}\right]}\end{array}$ \\
\hline \multirow[t]{4}{*}{ Walls } & brickwork & 0.10 & 2.856 & 0.35 \\
\hline & XPS & 0.08 & & \\
\hline & concrete block & 0.10 & & \\
\hline & gypsum plasterboard & 0.013 & & \\
\hline \multirow[t]{3}{*}{ Partitions } & gypsum plasterboard & 0.025 & 0.610 & 1.639 \\
\hline & air gap & 0.10 & & \\
\hline & gypsum plasterboard & 0.025 & & \\
\hline \multirow[t]{3}{*}{ Roof } & roofing felt & 0.005 & 6.24 & 0.16 \\
\hline & stone wool & 0.24 & & \\
\hline & clay tile & 0.025 & & \\
\hline \multirow[t]{4}{*}{ Ground floor } & concrete slab & 0.10 & 3.99 & 0.25 \\
\hline & XPS & 0.11 & & \\
\hline & fiberboard & 0.013 & & \\
\hline & asphalt & 0.09 & & \\
\hline
\end{tabular}

Table 3. Summary of the case study building main construction and thermal characteristics.

The dynamic simulation is performed both in free-floating and thermally-controlled conditions, for the purpose of the sensitivity analysis in terms of TDI and of the energy analysis in terms of IBE, respectively. Therefore, the simulation was performed in free-floating conditions i.e. with no operating HVAC and control system and no set point temperatures, in order to perform the TDI analysis. The thermally-controlled simulations were performed in order to carry out the energy analysis. Only in the thermally-controlled scenario the cooling system operates over the period June $1^{\text {st }}-$ September $30^{\text {th }}$, and the heating system operates every day over the period October $15^{\text {th }}-$ April $15^{\text {th }}$, according to the real building systems settings, occupancy schedule, and Italian climate zone prescriptions for residential buildings [51]. Setup air temperature is $25.5^{\circ} \mathrm{C}$ in summer and $21^{\circ} \mathrm{C}$ in winter, according to the local regulations for residential buildings in category $\mathrm{I}$, for 
living spaces in residential buildings, sedentary activity ( 1,2 met) and 1 and 0.5 clo in winter and summer, respectively [53]. All the data for the simulations have been assumed consistently with previous studies about Italian buildings' construction characteristics and technologies [54]. Nevertheless, the dynamic simulation tool is here used as a parametric analysis tool, with the aim not to predict the case study building thermalenergy performance in detail but to highlight and evaluate how such performance varies with respect to the variation of some significant key parameters.

\section{Results and Discussion}

\subsection{TDI analysis}

In order to assess the influence of input parameters on the performance of the case study, thermal year-round dynamic simulations are carried out by varying one input parameter at a time, in free-floating conditions. Results are post-processed in terms of TDI with reference to the whole building. Figure 2 shows the comparison between the calculated TDI values for each configuration, in both the evaluated scenarios, i.e. A and $\mathrm{N}$ configurations.

Fig. 2. TDI values with varying input parameters.

In general, in summer conditions, low opening percentage level is able to guarantee better TDI values both in the A and N configurations (A_Oa, N_Oa). Additionally, during summer the progressive decrease of the infiltration rate corresponds to the progressive increase of TDI in both configurations (A_Ia, N_Ia). In winter instead, a high opening percentage on the building façade positively affects the TDI in both configurations, given the higher solar gains entering the building (A_Oc, N_Oc,), with relatively lower benefit at higher percentage of opening rate due to higher thermal losses. Additionally, milder climate boundary conditions lead to the best values of TDI (A_Cc, N_Cc). Moreover, in winter conditions TDI decreases with increasing opening percentage on building façade and also infiltration rate for both configurations. This is due to the increased thermal losses generated by the presence of more openings and cracks on building façade. Furthermore, both A and $\mathrm{N}$ scenarios seem to be very sensitive to the climate parameter, since the TDI strongly decreases when shifting from a cold-temperate climate (i.e. Trieste) to a hot-temperate climate (i.e. Brindisi).

By considering the year-round analysis, peaks of TDI are found in association with the coolest climate weather file both in the A and N configurations (A_Ca, N_Ca). This means that extremely cold and windy climate boundary conditions are able to worsen the indoor thermal comfort level inside the building more than all the other considered input parameters. Additionally, a low opening percentage on the building façade 
(N_Oa, A_Oa) together with a low infiltration rate (N_Ia, A_Ia) can negatively affect year-round thermal performance of the control building both in the $\mathrm{A}$ and $\mathrm{N}$ configurations.

By analyzing the results from an Inter-Building Effect (IBE) perspective, the $\mathrm{N}$ configurations always present less mutually variable TDI values between winter and summer seasons, irrespective of the varying input parameters i.e. climate weather file, opening percentage, infiltration rate, and wind strength. Additionally, lower and higher TDI values are detected for the $\mathrm{N}$ configuration compared to the A configuration in summer and winter, respectively. This is imputable to the fact that, in the $\mathrm{N}$ configurations, the effect of outdoor boundary conditions on building indoor comfort and thermal performance is strongly mitigated by the presence of surrounding buildings, acting as "shields" from local boundary phenomena i.e. solar radiation and wind strength. This is true both in winter and summer conditions. Additionally, generally lower TDI values are found in winter, especially in the A configuration (i.e. Stand-alone). In particular, better TDI values are detected within the A configuration in winter, given the absence of surrounding buildings limiting the solar gains of the building. This is imputable to the IBE I phenomenon [24]. On the contrary, in summer conditions the $\mathrm{N}$ configuration provides better TDI due to the presence of the urban network of buildings acting as shading elements with respect to the strong incoming solar radiation. In the A configuration the TDI is better in winter rather than in summer. This is due to the total absence of adjacent buildings that makes the control building in the A configuration more sensitive to intense and durable solar radiation typical of summer days, which are characterized also by lower winds. This represents a benefit in winter and a penalty in summer, since it generates discomfort hours inside the building. In fact, summer months are characterized by less and weaker winds which are not able to mitigate the solar radiation effect in the A configuration. Finally, the A configuration is shown to be more affected to the climate IP rather than to the opening percentage IP. The configuration seems more sensitive to the local wind strength compared to the infiltration rate. Additionally, the $\mathrm{N}$ configuration seems to be positively affected by increasing opening percentage on building façade, and it is more sensitive to climate and infiltration rate rather than to the wind strength.

\subsection{Analysis of weather conditions}

The weather boundary conditions used for the simulations of the case study building are represented by the TMY files provided by the US Department of Energy [55]. In particular, the TMY weather file [55,56] of Trieste (Northern Italy), Pisa (Central Italy), and Brindisi (Southern Italy) were selected to be used in the simulation as representative of three different boundary conditions i.e. cold-temperate, temperate, and hottemperate conditions. Furthermore, these locations were chosen since the meteorological monitoring stations are all located at the same altitude above the sea level and with the same distance to the sea. Additionally, in order to isolate the wind effect, three further different weather files were generated by only varying the wind speed parameter starting from the TMY file for Pisa (central Italy), the city characterized by the mildest climate conditions both in terms of temperature and wind conditions. 
The purpose was to reproduce three weather boundaries characterized by different wind conditions i.e. strong $(\mathrm{Ca})$, medium $(\mathrm{Cb})$, and weak $(\mathrm{Cc})$ wind force, by keeping constant the same climate characteristics in term of all the other climate parameters i.e. global radiation, dry bulb temperature, relative humidity, etc. In this way, the effect of ventilation was isolated.

The maximum and average value of the wind intensity for the three selected climate weather files are summarized in Table 4. Figure 3 highlights the main differences between the selected weather TMY files in terms of wind speed. Additionally, the Pareto graphs in Figure 4 show the frequency distribution of the wind direction used in the simulation, which is the same for all weather files since only the wind intensity was modified.

\begin{tabular}{lcc}
\hline Climate weather file & \multicolumn{2}{c}{ Wind intensity [m/s] } \\
\cline { 2 - 3 } & Average & Maximum \\
\hline $\mathrm{Ca}$ & 4.9 & 37.0 \\
\hline $\mathrm{Cb}$ & 2.4 & 18.5 \\
\hline $\mathrm{Cc}$ & 1.2 & 9.3 \\
\hline
\end{tabular}

Table 4. Average and maximum values of wind speed in the newly generated TMY weather files.

Fig. 3. Wind intensity profile in each weather boundary condition i.e. $\mathrm{Ca}$ (strong wind), $\mathrm{Cb}$ (medium wind), Cc (weak wind).

Fig. 4. Pareto graphs with the wind direction distribution with decreasing order of frequency from the TMY weather files used for the simulation.

\subsection{Sensitivity analysis}

The sensitivity coefficients calculated for each of the considered parameters are summarized in Table 5.

\begin{tabular}{lcc}
\hline \multirow{1}{*}{ Input Parameters } & \multicolumn{2}{c}{ Sensitivity Coefficients } \\
\cline { 2 - 3 } & $\begin{array}{c}\text { Scenario 0 } \\
\text { Stand-alone building } \\
(A)\end{array}$ & $\begin{array}{c}\text { Scenario 1 } \\
\text { Network of buildings } \\
(N)\end{array}$ \\
\hline $\begin{array}{l}\text { C- Climate Weather file } \\
\text { [TMY] }\end{array}$ & 0.40 & 0.41 \\
\hline I- Infiltration rate [vol/h] & 0.09 & 0.38 \\
\hline O- Opening percentage [\%] & 0.27 & 0.42 \\
\hline
\end{tabular}


W- Wind strength [m/s] $0.09 \quad 0.05$

Table 5. Sensitivity coefficients calculated for the considered input parameters.

The orientation of the sensitivity analysis is that the higher the sensitivity coefficients, the stronger the effect of IPs on TDI variability. Additionally, the further the sensitivity coefficients deviate from a zero value, the higher the impact of the considered sensitivity parameter is on TDI compared to the others. But, focusing on the IBE analysis, the variation of sensitivity coefficients between $\mathrm{A}$ and $\mathrm{N}$ configuration shows the interbuilding role in affecting thermal behavior of buildings when considered within their real network, which is the main focus of the work.

In general, the climate weather file has the most significant role among the selected input parameters in affecting the TDI. Therefore, the climate boundary condition is found to be the most important parameter to take into account while predicting building thermal performance in dynamic simulation environment. This is confirmed by the fact that, in both scenarios, the choice of a different climate weather file leads to the biggest TDI variation when switching from the coldest climate to the hottest climate boundary condition. Nevertheless, even if the climate boundary condition has the strongest effect on indoor thermal comfort in buildings in general, the analysis of the results from an Inter-Building Effect perspective shows that there is only a weak perceivable difference (i.e. $2 \%$ ) between the two configurations, i.e. Stand-alone (A) and Network (N) scenarios, with respect to the climate boundary input.

Therefore, it is possible to assess that different boundary climate conditions are not able to significantly affect the Inter-Building Effect phenomenon. This is motivated by the fact that the IBE is a local scale phenomenon, which is more affected by parameters such as infiltration rate, opening percentage, and local wind effects rather than large-scale boundary conditions, i.e. climate conditions of the construction site. In fact, significant results from an IBE perspective are detected for the both opening percentage on building façade and infiltration rate. In particular, by shifting from the A to $\mathrm{N}$ configuration, a percentage difference of $36 \%$ and $76 \%$ is found in terms of TDI for the opening percentage on building façade and infiltration rate, respectively. This results show how Inter-Building Effect is a very significant phenomenon affecting both infiltration rate and opening percentage, which changes significantly between the Stand-alone building scenario (A) to the Network of buildings scenario (N). Additionally, a substantial difference, i.e. 51\%, is also detected between the $\mathrm{A}$ and $\mathrm{N}$ configurations with respect to the wind strength parameter.

Figure 5 summarizes the results of the seasonal (i.e. summer and winter) and year-round sensitivity analysis in free-floating conditions for each assessed scenario i.e. stand-alone building ("scenario 0") and network of buildings ("scenario 1").

Fig. 5(a-f). Results of the Sensitivity analysis of the different input parameters for scenario 0 (A-Standalone building) and scenario 1 (N-Network of buildings) in summer, winter, and year-round conditions. 
In winter and year-round analyses, the climate boundary conditions is the main parameter affecting the TDI in both configurations (i.e. A and N), since it is the more variable when shifting from low (cold) to high (hot). In particular, the year-round sensitivity analysis shows that the climate input parameter is followed by opening percentage on building façade, infiltration rate, and wind strength, in both scenarios (Figure 3c). The wind strength and the infiltration rate seem to have a similar lower effect from an IBE point of view. In summer, the wind strength is detected to have the most significant impact on thermal perception in terms of TDI in both scenarios when shifting from low to high. In winter, the climate boundary condition is shown to be once again the parameter mostly affecting the TDI while switching from cold to mild climate. Whereas, the wind strength impact on thermal comfort seems to be reduced.

Finally, from an Inter-Building Effect point of view, the infiltration rate is detected to be the most important parameter. In fact, it is the most variable parameter between the $\mathrm{N}$ scenario compared to the A scenario, especially when shifting from low (0.3) to medium (0.5). In particular, a variability of $76 \%$ is detected between the two configurations.

\subsection{Energy analysis}

In this section, the analysis of the primary energy consumption of the case study building in both A and $\mathrm{N}$ configurations is performed, by assuming a correlation between the sensitivity in terms of indoor comfort conditions (i.e. TDI) and building energy performance [41-44, 48,49]. The year-long dynamic simulation of the building energy requirement in terms of heating and cooling is carried out in the EnergyPlus simulation environment. The numerical analysis is performed by considering the following scenarios:

- Stand-alone building with high infiltration rate and opening percentage rate (A_max);

- Stand-alone building with low infiltration rate and opening percentage rate $\left(A \_m i n\right)$;

- Network of buildings with high infiltration rate and opening percentage rate $\left(N \_\right.$max $)$;

- Network of buildings with low infiltration rate and opening percentage rate $\left(N \_\right.$min).

Such scenarios represent the extreme boundary conditions in terms of the two parameters mainly affecting the IBE, i.e. infiltration rate and opening percentage on the building façade. All the simulations are performed in the mildest climate conditions, i.e. Pisa, because, as previously shown, weather condition is not the key boundary affecting the IBE. Additionally, each of the above mentioned scenarios is re-simulated without considering natural ventilation effect, and the IBE is re-calculated. The main purpose is to investigate and quantify the impact of wind on IBE. Figure 6 shows the primary energy requirement for heating and cooling $\left[\mathrm{kWh} / \mathrm{m}^{2}\right]$ for the case study "control" building in both the A and $\mathrm{N}$ configurations. 
Fig. 6. Primary energy requirement for heating and cooling for the case study "control" building in the A and $\mathrm{N}$ configuration, by considering natural ventilation.

First, the primary energy requirement for heating presents lower discrepancy between the two configurations than the primary energy requirements for cooling. This seasonal variation is not only due to the intensity of summer solar radiation, the mutual shading produced by the presence of close buildings, but also to the wind effect, which is detected to have a relatively higher impact in summer rather than in winter in terms of IBE. Additionally, the building global primary energy need is generally higher for the "max" scenarios compared to the "min" scenarios in both A and N configurations. This is true especially during summer, when the higher opening percentage on the façade and the higher infiltration rate make the building more affected by the high solar gains and the high thermal gain associated to the infiltration air volume. Furthermore, as expected, in both the "max" and "min" scenarios, non-negligible lower and higher primary energy need for cooling and heating, respectively, are detected for the control building when considered inside the network of surrounding buildings (i.e. $\mathrm{N}$ configuration). This is due to the fact that during summer the wind effect is lower and, additionally, the surrounding buildings are able to shade the solar radiation entering the building by reducing the solar gains and the peak cooling energy demand if compared to the Stand-alone configuration (A), as observed in the first IBE analyses [24]. In particular, a maximum reduction of $34 \%$ and $42 \%$ of the primary energy demand for cooling is detected between the $\mathrm{N}$ and A configuration for the max and $\min$ scenarios, respectively. In winter, the presence of the urban built environment generates slightly higher heating demand ( $44 \%$ and $48 \%$ ) in both scenarios i.e. $\max$ and min, respectively, due to the less thermal gain of the control building thanks to the shading action of adjacent surrounding buildings. Such results are consistent with IBE analyses previously carried out on a different case study by the same authors [25].

Consistent results are found for the same scenarios simulated without considering the impact of natural ventilation on building global primary energy demand. Such results are displayed in Figure 7 in terms of primary energy requirement for heating and cooling $\left[\mathrm{kWh} / \mathrm{m}^{2}\right]$ for the case study "control" building, in both $\mathrm{A}$ and $\mathrm{N}$ configuration.

Fig. 7. Primary energy requirement for heating and cooling for the case study "control" building in the A and $\mathrm{N}$ configurations, without taking into account natural ventilation phenomena.

The wind effect in the different evaluated scenarios i.e. A and $\mathrm{N}$ is highlighted in Figure 8, which reports the difference [\%] in the primary energy requirement for heating and cooling between the assessed scenarios attributable to natural ventilation. 
Figure 8. Monthly difference in terms of primary energy requirement [\%] with and without accounting for natural ventilation, for all the assessed scenarios.

In general, the primary energy difference [\%] between the scenarios simulated with and without accounting for natural ventilation is higher in winter rather than in summer, when the discrepancies are detected to be lower. This is due to the fact that in summer the wind is weaker with respect to winter months, therefore the impact of the natural ventilation on building energy performance is less evident in the considered case study locations. By comparing the only $\max$ and $\min$ scenarios irrespective of the urban configuration, i.e. $A \_\min$ vs $A \_$max and $N \_$min vs $N \_$max , it is possible to isolate the impact of natural ventilation on building energy performance. In particular, the higher the difference between the max and $\min$ scenarios, the higher the effect of natural ventilation. In this perspective, the maximum difference between max and min scenarios is found in March $(\sim 58 \%)$ and April $(\sim 27 \%)$ for the A and N configurations, respectively. Such discrepancies represent the impact of wind effect in the accuracy of the energy prediction carried out by means of dynamic simulation tools. Such results are consistent with previous studies assessing the impact of natural ventilation on building thermal-energy performance [57-59].

Therefore, by comparing the $\mathrm{A}$ and $\mathrm{N}$ scenarios, i.e. $A \_$min vs. $N \_$min and $A \_m a x$ vs. $N \_m a x$, the IBE phenomenon can be isolated in order to understand its relationship with natural ventilation and wind effect. In particular, Figure 6 shows how the primary energy difference [\%] between the simulations carried out with and without accounting for natural ventilation is higher in the $\mathrm{N}$ configuration in winter months and is slightly higher in the A configuration in spring-summer months. This means that, during summer, when winds are typically lower, the wind effect on building energy performance is not so impactful, and the small energy gap detected with respect to the $\mathrm{N}$ configuration is totally attributable to the IBE. In winter by contrast, the presence of the wind is non negligible and therefore must be taken into account. This is true especially in the $\mathrm{N}$ configuration, where the impact of the other meteorological boundary agents is weaker with respect to the wind effect due to the IBE. In fact, in the $\mathrm{N}$ configuration, the winter wind effect on building performance seems to be stronger due to the fact that the IBE makes the other environmental forcing, e.g. solar radiation, less impactive.

The maximum primary energy gap between $\mathrm{A}$ and $\mathrm{N}$ configurations is found to be about $22 \%$ (October) and $41 \%$ (March), for the $\max$ and $\min$ scenarios, respectively.

A graphic representation of the difference between the two configurations i.e. A and $\mathrm{N}$ in terms of Interbuilding Effect, with and without taking into account natural ventilation, is provided in Figure 9.

Fig. 9. Inter-Building Effect comparison between the different scenarios assessed. 
A maximum IBE of $77 \%$ and $24 \%$ is detected for the $\max$ scenario and of $94 \%$ and $25 \%$ for the min scenario, in winter and summer, respectively, with considering natural ventilation. The same analysis without considering wind effect leads to maximum IBE values of $114 \%$ and $26 \%$ for the max scenario and of $172 \%$ and $30 \%$ in the $\min$ scenario, for winter and summer, respectively. Therefore, the IBE analysis shows how, for all the assessed scenarios, an over-estimation and under-estimation of the primary energy consumption for cooling and heating, respectively, results when predicting building energy performance without taking into account the surrounding urban context. Generally, higher values of IBE are found for the scenarios simulated without taking into account natural ventilation if compared to the realistic scenarios, i.e. with natural ventilation. This means that, unlike the studies previously carried out about IBE, the additional parameter taken into account into the present analysis i.e. natural ventilation generates a decrease of the IBE due to its "mixing" effect. While previous studies demonstrated how the additional considered parameters e.g. lighting had the effect of increasing the IBE, in the present case study the additional parameter taken into account, e.g. natural ventilation, reduces the IBE, given its damping and mitigation effect through a "mixing" action with respect to all the other environmental parameters and phenomena.

Moreover, the results show slightly smaller differences in terms of IBE during summer between the simulations carried out with and without considering natural ventilation phenomena, in both $\max$ and $\min$ scenarios. In particular, a maximum difference of $5 \%$ and $2 \%$ between IBE values is detected in summer for min and max scenarios, respectively. This is probably related to the lower winds in summer. In winter, such difference reaches up to $79 \%$ and $37 \%$ for min and $\max$ scenarios, respectively. Therefore, in winter months the differences in terms of IBE attributable to only wind effects are considerably higher and non-negligible. The year-round average difference between the assessed case scenarios simulated with and without accounting for wind effects is detected to be about $12 \%$ and $6 \%$ for the min and max scenarios, respectively.

\section{Conclusions}

In this paper, the evaluation of the impact of natural ventilation on the Inter-Building Effect (IBE) in terms of building primary energy requirement was performed. While previous studies focused on the ventilation performance prediction in terms of improvement of buildings' indoor thermal comfort and on the cooling capability of differently natural ventilation-based passive strategies, especially in the context of office buildings, the innovative contribution of the present work consisted of the elaboration of a new methodology to determine the role of natural ventilation while predicting building energy requirement in a dynamic simulation environment, based on a preliminary sensitivity analysis and numerical analysis. In particular, the role of wind and airflow rates through building openings and around buildings in an urban environment were assessed for its effect on building energy demand for heating and cooling through an IBE perspective. To this purpose, a four story residential building representative of the Italian building stock and its suburban neighborhood were selected. Additionally, (i) Climate boundary conditions, (ii) infiltration rates, (iii) opening 
percentages on building façade, and (iv) wind strength were selected as varying key input parameters affecting the building indoor thermal conditions in terms of Thermal Deviation Index. Different scenarios were simulated in order to determine the IBE variation directly attributable to the natural ventilation phenomena. The simulation results establish that (i) building geometry and surroundings played a major role in determining wind effect, and (ii) the impact of natural ventilation with respect to the IBE phenomenon in terms of building primary energy requirement for heating and cooling was non-negligible. In fact, the results of the sensitivity analysis in terms of IBE showed that the opening percentage and the infiltration rate are the input parameters the mostly affect TDI (i.e. $36 \%$ and $76 \%$, respectively), followed by the wind strength (i.e. $51 \%$ ). The climate boundary conditions seemed not to have a considerable impact in terms of IBE. This is attributable to the fact that IBE is a local phenomenon therefore not much influenced by "global" or largerscale boundary conditions. In fact, IBE is much more dependent on the specific configuration of the urban environment considered in terms of shape, orientation, opening percentage, and building features in general rather than on climate variability. The numerical analysis showed that, unlike previous studies, the additional parameter taken into account in the present analysis, i.e. natural ventilation, generates a decrease of the IBE. This is attributable to its "mixing" effect. In fact, higher values of IBE were found for the scenarios simulated without taking into account natural ventilation if compared to the realistic scenarios, i.e. with natural ventilation. In particular, a maximum additional contribution to the IBE of $2 \%$ and $37 \%$ in summer, and of $5 \%$ and $79 \%$ in winter, for the $\max$ and $\min$ assessed scenarios, respectively, was directly attributable to natural ventilation phenomena. Therefore, wind effect represented a non-negligible phenomenon having a significant impact on a local Inter-Building scale, and it needs to be considered while performing building energy prediction since it is significantly affected by both single-building and urban configuration.

Consistent with the results of previous related studies, the IBE has again been shown to meaningfully impact the accuracy of building energy performance prediction. Here we demonstrated that building energy performance prediction strongly depends on the configuration of the urban environment affecting local ventilative phenomena. The inter-building scale cannot be neglected in thermal-energy assessment of new and existing buildings located in an urban context. These results demonstrate that natural ventilation needs to be taken into account when applying the IBE approach to more realistically model and predict building energy performance. More broadly, these results demonstrate the need for further research into neighborhood and urban scale phenomena that may meaningfully impact the energy performance predictions of buildings in cities.

\section{Acknowledgements}

The authors' acknowledgments are due to H2CU (Honors Center of Italian Universities) for supporting the international cooperation among the authors. Taylor's contribution to the work was supported by the National Science Foundation under Grant No. 1142379. Any opinions, findings, and conclusions or 
recommendations expressed in this material are those of the authors and do not necessarily reflect the view of the National Science Foundation or H2CU. The work is partially funded by the Spanish government (ENE2011-22722) and by the Fondazione Cassa di Risparmio di Perugia within the framework of the project UMBRA ARTIS (cod. 201.0223.021). A.L. Pisello's acknowledgments are due to the "CIRIAF program for UNESCO" in the framework of the UNESCO Chair "Water Resources Management and Culture", for supporting her research.

\section{References}

[1] Polydoros, A, Cartalis, C, Use of Earth Observation based indices for the monitoring of built-up area features and dynamics in support of urban energy studies, Energy and Buildings 98 (2015) 92-99, http://dx.doi.org/10.1016/j.enbuild.2014.09.060.

[2] Santamouris, M, Analyzing the heat island magnitude and characteristics in one hundred Asian and Australian cities and regions, Science of the Total Environment Volume 512-513 (2015) 582-598.

[3] Taha, H, Akbari, H, Rosenfeld, A, Huang, J, Residential cooling loads and the urban heat island - the effects of albedo, Building and Environment, 23, Issue 4, (1988), 271-283.

[4] Natarajan, S, Rodriguez, J, Vellei, M, A filed study of indoor thermal comfort in the subtropical highland of Bogota, Colombia, Journal of Building engineering $4 \quad$ (2015) 237-246. http://dx.doi.org/10.1016/j.enbuild.2015.10.003.

[5] Perez-Lombard, L, Ortiz, J, Pout, C, A review on buildings energy consumption information Energy and Buildings 40 (2008) 394-398.

[6] International Energy Agency, Key World Energy Statistics, 2006.

[7] Shi, W, Wang, S, Yang, Q, Climate change and global warming Rev Environ Sci Biotechnol (2010) 9:99102 DOI 10.1007/s11157-010-9206-7.

[8] Houghton, JT, Jenkins, GJ, Ephraums, JJ, Climate change: the IPCC assessment, , Cambridge University press.

[9] Santamouris, M, Cartalis, C, Synnefa, A, Local urban warming, possible impacts and a resilience plan to climate change for the historical center of Athens, Greece, , Sustainable Cities and Society. Available online 19 February 2015, In Press, Corrected Proof, doi:10.1016/j.scs.2015.02.001.

[10] Santamouris, M, Using cool pavements as a mitigation strategy to fight urban heat island-A review of the actual developments, Renewable and Sustainable Energy Reviews, Volume 26, October 2013, Pages 224 240, doi:10.1016/j.rser.2013.05.047.

[11] Dimoudi, A, Nikopoulou, M, Vegetation in urban environment: microclimate analysis and benefits, Energy and Buildings, 35 (2003), 69-76. 
[12] Huang, YJ, Akbari, H, Taha, H, Rosenfeld, AH, 1987: The Potential of Vegetation in Reducing Summer Cooling Loads in Residential Buildings. J. Climate Appl. Meteor., 26, 1103-1116 (1987). doi: http://dx.doi.org/10.1175/1520-0450.

[13] Oropeza-Perez, I, Østergaard, PA, Potential of natural ventilation in temperate countries - A case study of Denmark Applied Energy 114 (2014) 520-530. doi.org/10.1016/j.apenergy.2013.10.008.

[14] Ai, ZT, Mak, CM, From street canyons microclimate to indoor environmental quality in naturally ventilated urban buildings: issues and possibilities for improvement, Building and Environment 94 (2015) 489-503. http://dx.doi.org/10.1016/j.enbuild.2015.10.008.

[15] Kolokotroni, M, Aronis, A, Cooling-energy reduction in air-conditioned offices by using night ventilation Applied Energy 63 (1999) 241-253.

[16] Artmann, N, Manz, H, Heiselberg, P, Climatic potential for passive cooling of buildings by night-time ventilation in Europe .

[17] Martins, NR, De Graca, GC, Validation of numerical simulation tools for wind-driven natural ventilation design, Building simulations 9 (2016) 75-87. doi 10.1007/s12273-015-0251-6.

[18] Chiesa, G, Grosso, M, Geo-climatic applicability of natural ventilative cooling in the Mediterranean area, Energy and Buildings 107 (2015) 376-391. http://dx.doi.org/10.1016/j.enbuild.2015.08.043.

[19] Barbosa, S, Southall, KIR, Thermal comfort in naturally ventilated buildings with double skin façade under tropical climate conditions: the influence of key design parameters, Energy and Buildings, 109 (2015) 397 406. http://dx.doi.org/10.1016/j.enbuild.2015.10.029

[20] Andelkovic, AS, Gvozdenac-Urosevic, B, Kljajic, M, Ignjatovic, MG, Experimental research of the thermal characteristics of a mutli-stroey naturally ventilated double-skin façade, Energy and Buildings 86 (2015) 766781.

[21] Faggianelli, GA, Brun, A, Wurtz, E, Muselli, M, Assessment of different airflow modeling approaches on a naturally ventilated mediterranean building, Energy and Buildings, 107 (2015) 345-354. http://dx.doi.org/10.1016/j.enbuild.2015.08.038

[22] Givoni, B, Comfort, climate analysis and building design guidelines, Energy and Buildings, Volume 18, Issue 1, 1992, Pages 11-23.

[23] Shaviv, E, Yezioro, A, Capeluto, IG, Thermal mass and night ventilation as passive cooling design strategy, Renewable Energy, Volume 24, Issues 3-4, November 2001, Pages 445-452.

[24] Pisello, AL, Taylor, Xu, X, Cotana, F, Inter-building effect: Simulating the impact of a network of buildings on the accuracy of building energy performance predictions, Building and Environment 58 (2012) 37-45.

[25] Pisello, AL, Taylor, JE, Cotana, F, Simulating the effect of urban morphology on indoor thermal behavior: An Italian case study, Proceedings of the 2013 Winter Simulation Conference, 2013, Pages 2008-2019,

[26] Pisello, AL, Castaldo, VL, Poli, T, Cotana, F, Simulating the Thermal-Energy Performance of Buildings at the Urban Scale: Evaluation of Inter-Building Effects in Different Urban Configurations, Journal of Urban Technology, Volume 21, Issue 1, January 2014, Pages 3-20, 
[27] Pisello, AL, Castaldo, VL, Taylor, JE, Cotana, F, Energy and Buildings, Volume 76, June 2014, Pages 513 523, Expanding Inter-Building Effect modeling to examine primary energy for lighting,

[28] Han, Y, Taylor, J, Pisello, A, Toward Mitigating Urban Heat Island Effects: Investigating the ThermalEnergy Impact of Bio-Inspired Retro-reflective Building Envelopes in Dense Urban Settings, Energy and Buildings, 102 (2015) 380-389.

[29] Liua, J, Heidarinejad, M, Gracik, S, Srebric, J, The impact of exterior surface convective heat transfer coefficients on the building energy consumption in urban neighborhoods with different plan area densities, Energy and Buildings 86 (2015) 449-463, http://dx.doi.org/10.1016/j.enbuild.2014.10.062.

[30] Jamaludina, AA, Hussein, H, Ariffin, A, Keumala, N, A study on different natural ventilation approaches at a residentialcollege building with the internal courtyard arrangement, , Energy and Buildings 72 (2014) 340352, http://dx.doi.org/10.1016/j.enbuild.2013.12.050.

[31] Ramponi, R, Gaetani, I, Angelotti, A, Energy and Buildings, Volume 78, August 2014, Pages 24-34, Influence of the urban environment on the effectiveness of natural night-ventilation of an office building,

[32] Ramponi, R, Blocken, BJE, CFD simulation of cross-ventilation for a generic isolated building: impact of computational parameters. Building and Environment, 53, (2012), 34-48.

[33] Ramponi, R, Blocken, BJE, CFD simulation of cross-ventilation flow for different isolated building configurations: validation with wind tunnel measurements and analysis of physical and numerical diffusion effects. Journal of Wind Engineering and Industrial Aerodynamics, 104-106 (2012) 408-418.

[34] Blocken, B, Defraeye, T, Derome, D, Carmeliet, J, High-resolution CFD simulations for forced convective heat transfer coefficients at the facade of a low-rise building, Building and Environment 44 (2009) 23962412.

[35] Liu, J, Srebric, J, Yu, N, Numerical simulation of convective heat transfer coefficients at the external surfaces of building arrays immersed in a turbulent boundary layer, International Journal of Heat and Mass Transfer 61 (2013) 209-225.

[36] Defraeye, T, Carmeliet, J, A methodology to assess the influence of local wind conditions and building orientation on the convective heat transfer at building surfaces Environmental Modelling \& Software 25 (2010) 1813-1824.

[37] Huang, J, Winkelmann, F, Buhl, F, Linking the comis multi-zone airflow model with the energyplus building energy simulation program, Lawrence Berkeley National Laboratory, Berkeley CA, USA

[38] Pedersen, C, Fisher, D, Liesen, R, Taylor, R, Strand, R, The Building Systems Laboratory, University of Illinois, Urbana IL, USA Drury Crawley, US Department of Energy, Washington DC, USA Linda Lawrie, US Army Corps of Engineers, Construction Engineering Research Laboratory Champaign IL, USA

[39] Hensen, J, Lamberts, R, Building Performance Simulation for Design and Operation Hardcover - February 24, 2011, by.

[40] Pisello, AL, Piselli, C, Cotana, F, Influence of human behavior on cool roof effect for summer cooling, Building and Environment (2014), doi: 10.1016/j.buildenv.2014.09.025. 
[41] Crawley, DB, Lawrie, LK, Winkelmann, FC, Buhl, WF, Joe Huang, Y, Pedersen, CO, Strand, RK, Liesen, RJ, Fisher, DE, Witte, MJ, Glazer, J, EnergyPlus: creating a new-generation building energy simulation program Energy and Buildings, 33, 4, 443-457.

[42] Pisello, AL, Goretti, M, Cotana, F, A method for assessing buildings' energy efficiency by dynamic simulation and experimental activity, Applied Energy 97 (2012) 419-429.

[43] UNI TS 11300-1: Determinazione del fabbisogno di energia termica dell'edificio per la climatizzazione estiva ed invernale (italian)

[44] EN 15251:2007, Indoor environmental input parameters for design and assessment of energy performance of buildings addressing indoor air quality, thermal environment, lighting and acoustics, 2007.

[45] Ballarini, I, Corrado, V, Application of energy rating methods to the existing building stock: Analysis of some residential buildings in Turin, Energy and Buildings 41 (2009) 790-800. doi:10.1016/j.enbuild.2009.02.009.

[46] Tronchin, L, Fabbri, K, Energy performance building evaluation in Mediterranean countries: Comparison between software simulations and operating rating simulation, Energy and Buildings 40 (2008) 1176-1187, doi:10.1016/j.enbuild.2007.10.012.

[47] Caldera, M, Corgnati, SP, Filippi, M, Energy demand for space heating through a statistical approach: application to residential buildings, Energy and Buildings 40 (2008) 1972-1983, doi:10.1016/j.enbuild.2008.05.005.

[48] UNI EN 15242:2008: Ventilazione degli edifici - Metodi di calcolo per la determinazione delle portate d'aria negli edifici, comprese le infiltrazioni (italian)

[49] UNI 10339:1995, Air conditioning systems for thermal comfort in buildings, Generals, classifications, and requirements, Offer, orders, and supply classifications.

[50] Pisello, AL, Goretti, M, Cotana, F, Building energy efficiency assessment by integrated strategies: dynamic simulation, sensitivity analysis and experimental activity, Third International Conference on Applied Energy - 16-18 May 2011 - Perugia, Italy.

[51] Lam, JC, Hui, SCM, Sensitivity analysis of energy performance of office buildings. Building and Environment 31 (1996) 27-39.

[52] Pisello, AL, Asdrubali, F, Human-based energy retrofits in residential buildings: A cost-effective alternative to traditional physical strategies, Applied Energy $133 \quad$ (2014) 224-235, http://dx.doi.org/10.1016/j.apenergy.2014.07.049

[53] EN 15251:2006, Indoor environmental input parameters for design and assessment of energy performance of buildings addressing indoor air quality, thermal environment, lighting and acoustics.

[54] Typology Approach for Building stock energy Assessment, 2009-2012, Intelligent Energy Europe.

[55] http://apps1.eere.energy.gov/buildings/energyplus/cfm/weather_data3.cfm/region=6_europe_wmo_region_ $6 /$ country=ITA/cname=Italy 
[56] Chan, ALS, Developing a modified typical meteorological year weather file for Hong Kong taking into account the urban heat island effect, Building and Environment 46 (2011) 2434-2441. doi:10.1016/j.buildenv.2011.04.038

[57] Rijal, HB, Tuohy, P, Humpreys, MA; Nicol, JF, Samuel, A, Clarke, J, Using results from field surveys to predict the effect of open windows on thermal comfort and energy use in buildings, Energy and Buildings 39 (2007) 823-836.

[58] Santamouris, M, Papanikolaou, N, Livada, I, Koronakis, I, Georgakis, C, Arigriou, A, Assimakopoulos, DN, On the impact of urban climate on the energy consumption of buildings, Solar Energy 70-3 (2001) 201.216

[59] Kolokotroni, M, Giannitsaris, I, R. Watkins, R, The effect of the London urban heat island on building summer cooling demand and night ventilation strategies, Solar Energy 80 (2006) 383-392. doi:10.1016/j.solener.2005.03.010 
Figure 1

a)

b)
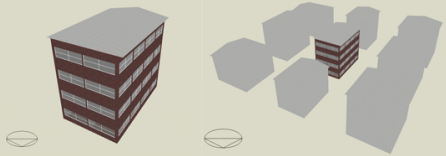


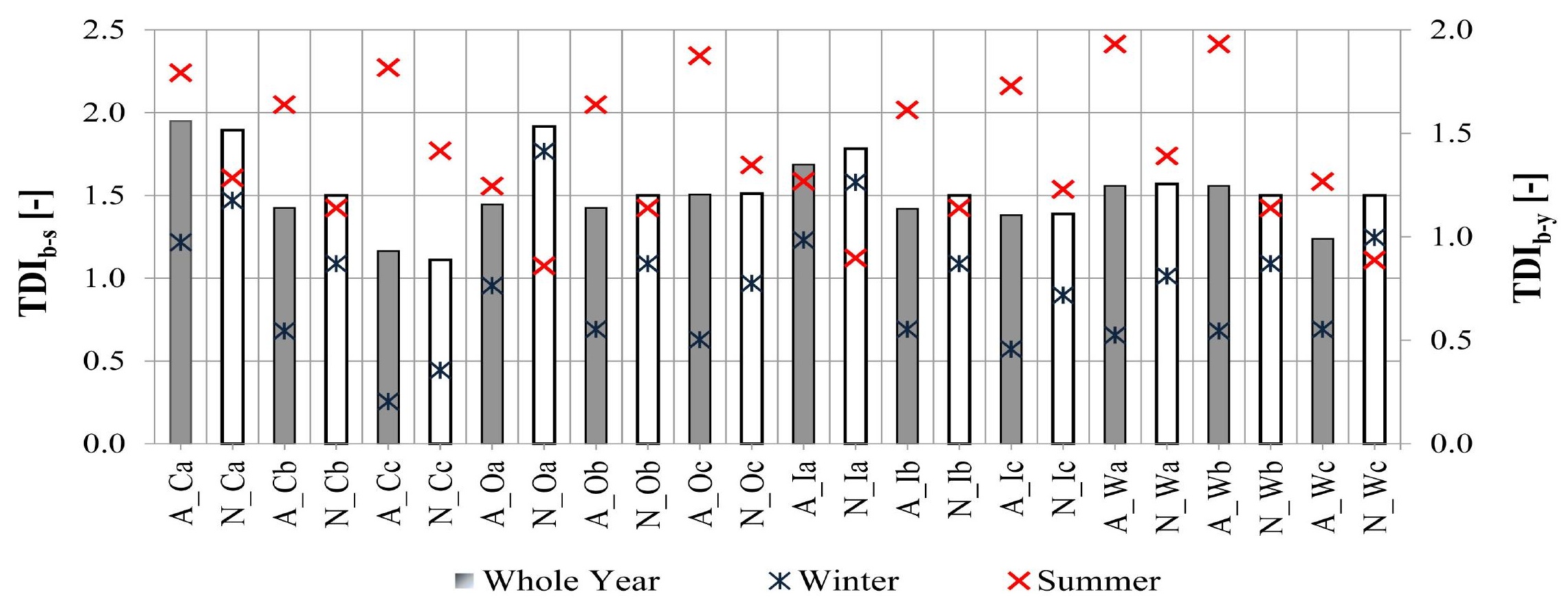


Figure 3

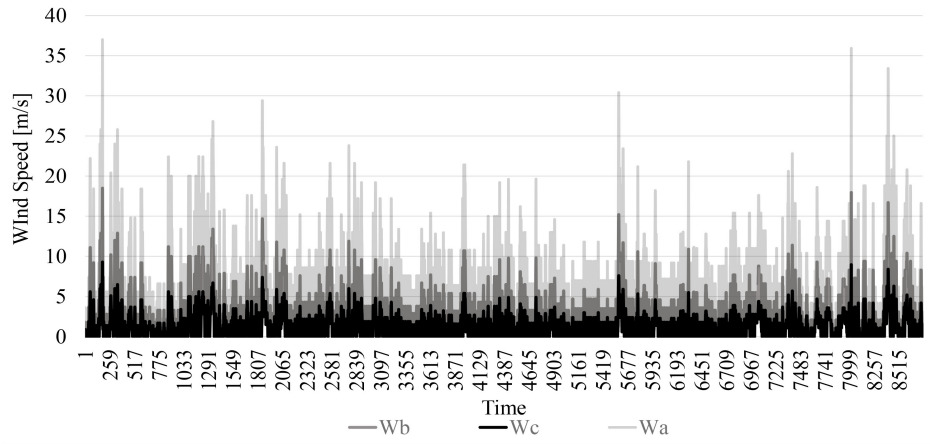


Figure 4

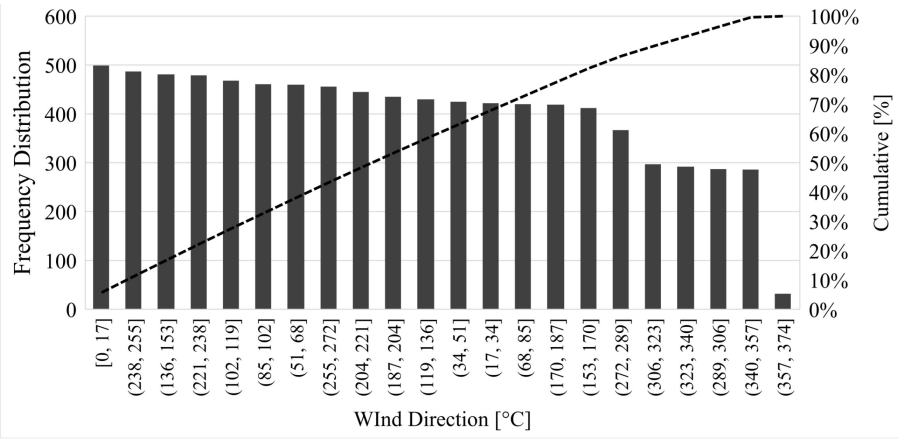



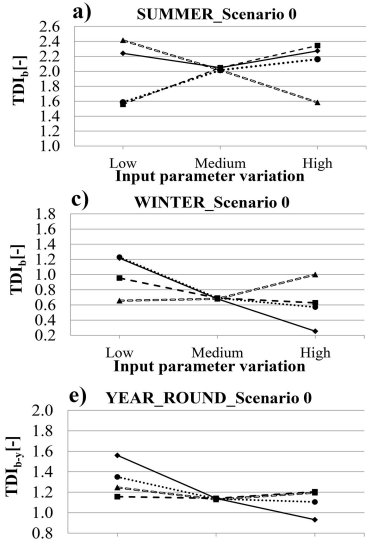

e) YEAR_ROUND_Scenario 0

Low Medium High Input parameter variation

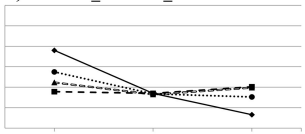

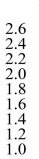

b) SUMMER_Scenario 1
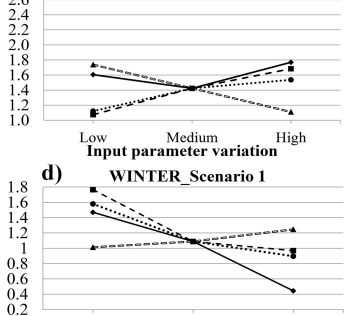

Low Medium High Input parameter variation
1.8

1.6

1.4

1.2

1.0

0.8

d) f) YEAR_ROUND_Scenario 1

\section{WINTER_Scenario 1}

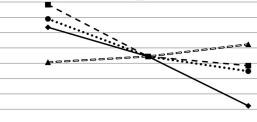

Low Medium High Input parameter variation

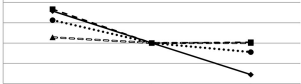

Low Medium High

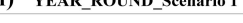

$\rightarrow$ Climate weather file - - Infiltration rate $\cdots \bullet \cdot$ Opening percentage $-\star-$ Wind strenght 
Figure 6

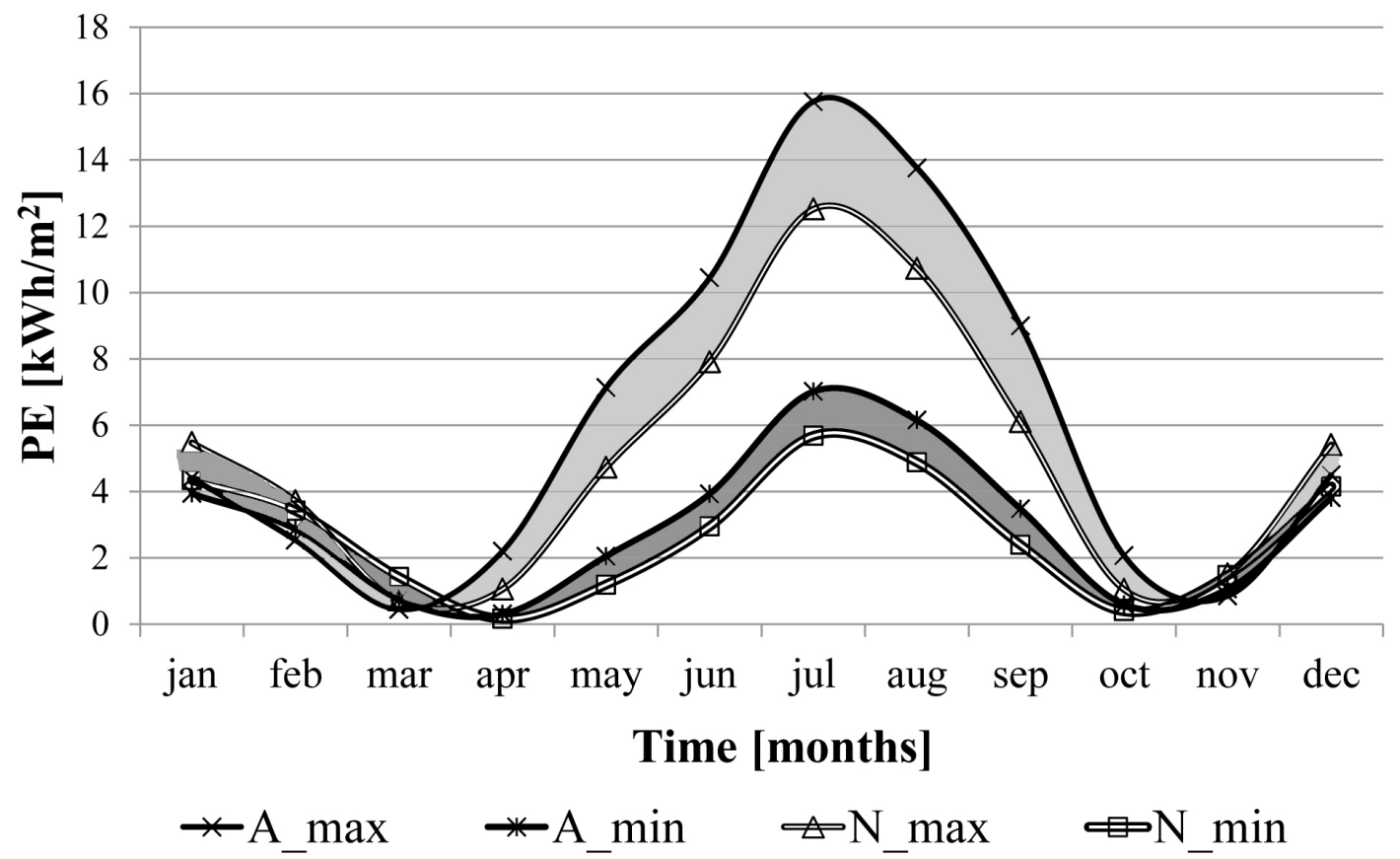




\section{Figure 7}

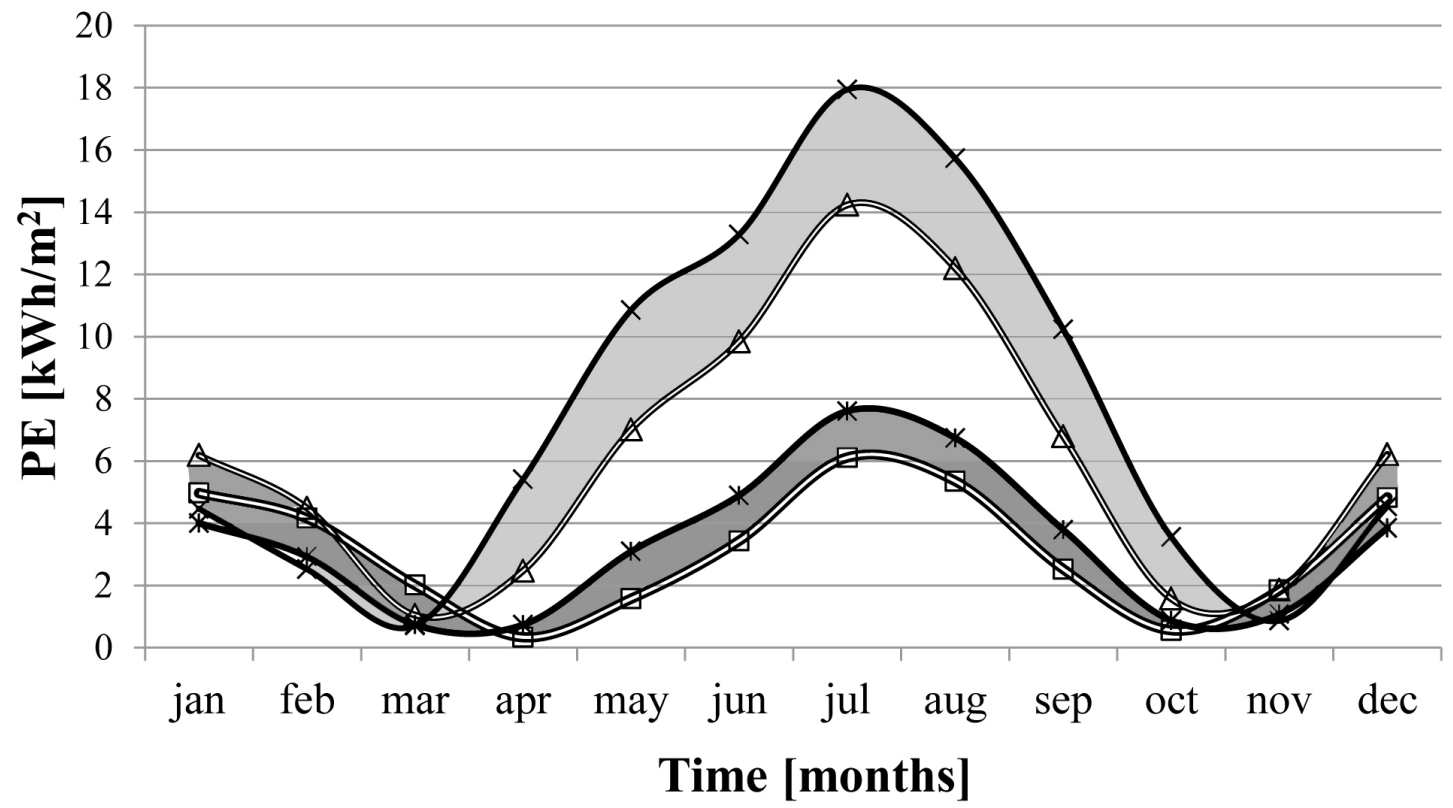

*A_max $\quad *$ A_min $\triangle$ N_max $\square$ N_min 


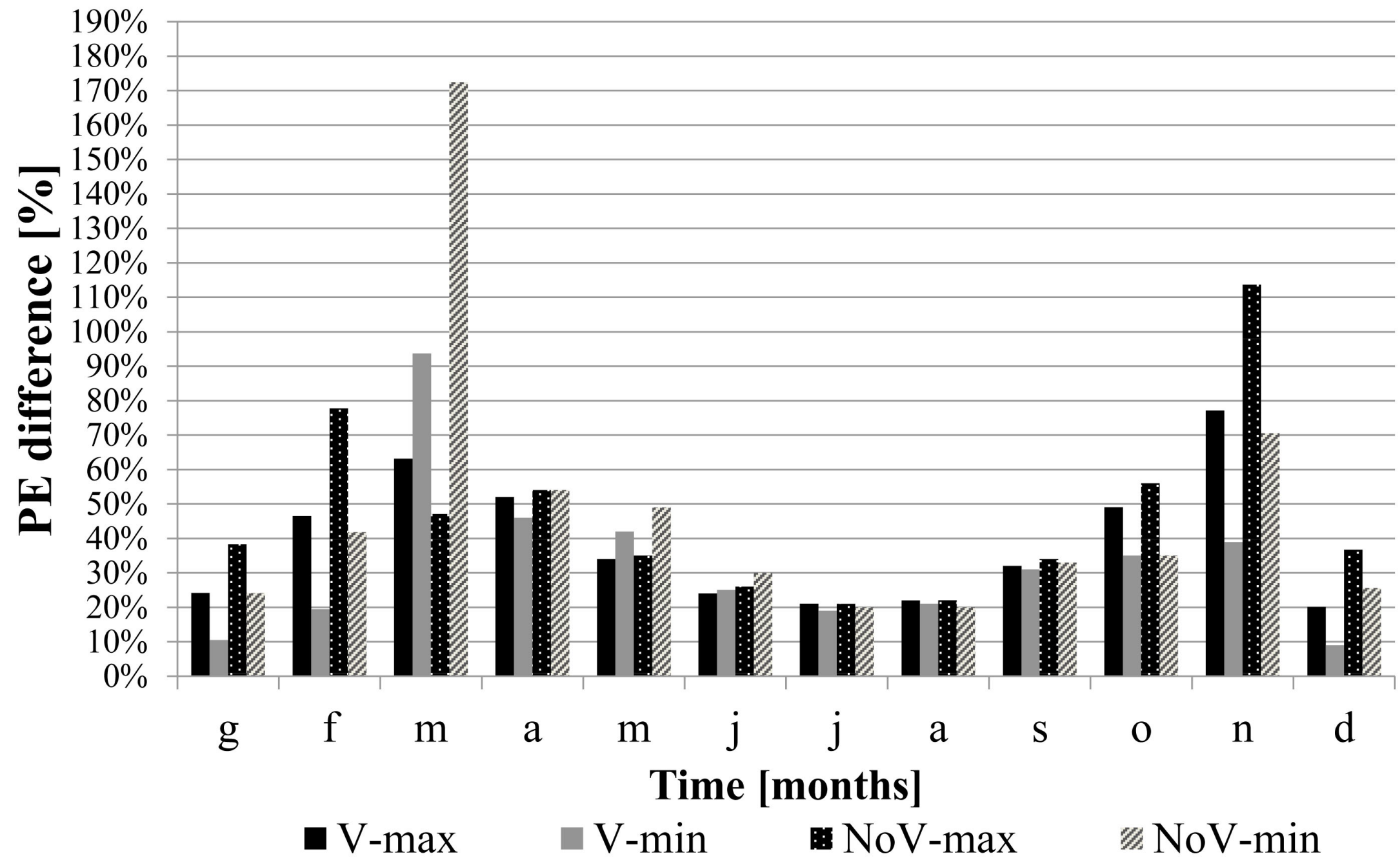




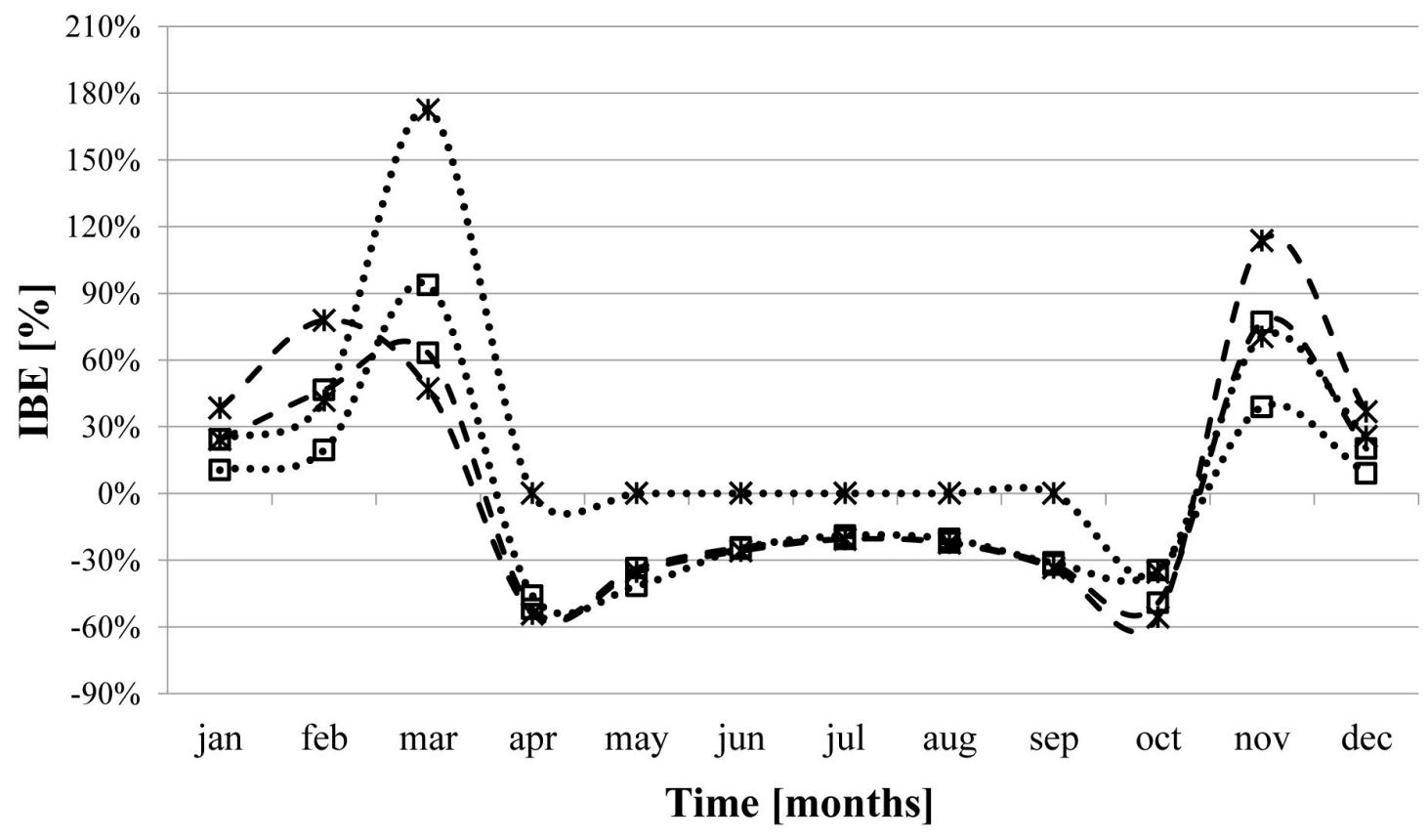

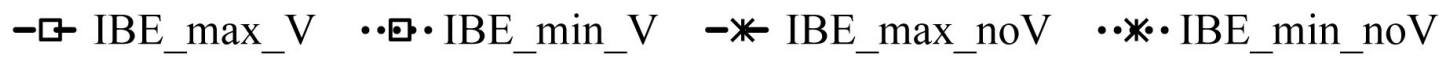

